\title{
Sufficient and Necessary Conditions for the Classification of Sturm-Liouville Differential Equations with Complex Coefficients
}

\author{
Bing Xie and Jian Gang Qi \\ Department of Mathematics, Shandong University at Weihai, Weihai 264209, China \\ Correspondence should be addressed to Jian Gang Qi, qjg816@163.com \\ Received 23 February 2011; Accepted 27 April 2011 \\ Academic Editor: Nicholas D. Alikakos
}

Copyright (C) 2011 B. Xie and J. G. Qi. This is an open access article distributed under the Creative Commons Attribution License, which permits unrestricted use, distribution, and reproduction in any medium, provided the original work is properly cited.

This paper gives sufficient and necessary conditions for the classification of Sturm-Liouville differential equations with complex coefficients given by Brown et al. These conditions involve weighted Sobolev subspaces and the asymptotic behavior of elements in the maximal domain. The results of the present paper generalize the corresponding results for formally symmetric SturmLiouville differential equations to non-self-adjoint cases.

\section{Introduction}

Consider the Sturm-Liouville differential expression

$$
\tau y:=w^{-1}\left[-\left(p y^{\prime}\right)^{\prime}+q y\right]=\lambda y \quad \text { on }[a, b),
$$

where $p, q$ are both complex valued, $w(x)$ is a positive weight function, $-\infty<a<b \leq+\infty$, and $\lambda$ is the so-called spectral parameter. We call $\tau$ a formally symmetric differential expression if $p, q$ are both real valued; otherwise $\tau$ is called formally nonsymmetric. In all cases, we call $\tau$ a formally differential expression or operator.

Let $L_{w}^{2}$ denote the Hilbert space

$$
L_{w}^{2}:=\left\{y \text { is measurable }:[a, b) \longrightarrow \mathbb{C}: \int_{a}^{b} w(x)|y(x)|^{2} \mathrm{~d} x<\infty\right\}
$$


with inner product $\langle y, z\rangle:=\int_{a}^{b} \bar{z}(x) w(x) y(x) \mathrm{d} x$ and the norm $\|y\|^{2}=\langle y, y\rangle$ for $y, z \in L_{w}^{2}$. We call a solution $y$ of (1.1) an $L_{w}^{2}$-solution or square integrable solution if $y \in L_{w}^{2}$. Set

$$
\Phi(\tau)=\left\{y \in L_{w}^{2}: y, p y^{\prime} \in \mathrm{AC}_{\mathrm{loc}}, \tau y \in L_{w}^{2}\right\}
$$

where $\mathrm{AC}_{\mathrm{loc}}=\mathrm{AC}_{\mathrm{loc}}([a, b), \mathbb{C})$ is the set of complex valued functions that are absolutely continuous on each compact subinterval of $[a, b)$. We call $\Phi(\tau)$ the natural (or maximal) domain associated with the formally differential operator $\tau$.

The aim of the present paper is to study the asymptotic behavior of elements of $\Phi(\tau)$. This is closely related to the classification of (1.1) according to the number of square integrable solutions of (1.1) in suitable weighted integrable spaces. The study of this problem has a long history started with the pioneering work of Weyl in 1910 [1]. When $p(x)$ and $q(x)$ are all real valued, Weyl classified (1.1) into the limit point and limit circle cases in the geometric point of view by introducing the $m(\lambda)$-functions, where we say that $\tau$ or (1.1) is in the limit point case at $b$ if there exists exactly one $L_{w}^{2}$-solution (up to constant multiple) for $\lambda \in \mathbb{C}$ with $\operatorname{Im} \lambda \neq 0$ and is in the limit circle case if all solutions belong to $L_{w}^{2}$ for $\lambda \in \mathbb{C}$ with $\operatorname{Im} \lambda \neq 0$. This work has been greatly developed and generalized to formally symmetric higher-order differential equations and Hamiltonian differential systems. For this line, the reader is referred to [2-10] and references therein.

The same problem was also studied by Sims in 1957 for the case where $q(x)$ is complex valued [11]. He considered the case where $p(x)=w(x) \equiv 1$ and $\operatorname{Im} q(x)$ is semibounded and classified (1.1) into three cases. Recently, this work has been extensively generalized by Brown et al. [12] under mild assumptions on weighted function $w(x)$ and the complex valued coefficients $p(x), q(x)$. They proved that there exist three distinct possible cases for (1.1).

For formally symmetric $\tau$, it is well known (see $[13,14])$ that $(1.1)$ is in the limit point case at $b$ if and only if

$$
p(x)\left[y_{2}(x) \overline{y_{1}^{\prime}}(x)-\overline{y_{1}}(x) y_{2}^{\prime}(x)\right] \longrightarrow 0 \quad \text { as } x \longrightarrow b
$$

for $y_{1}, y_{2} \in \Phi(\tau)$. This kind of characterization (1.4) plays an important role in spectral theory of differential operators since (1.4) gives a natural boundary condition of functions in $\Phi(\tau)$ at the end point $b$. In this case every self-adjoint extension associated with the differential expression needs not a boundary condition at $b$. The analogues of the result (1.4) are also valid for both formally symmetric higher-order differential equations and Hamiltonian differential systems (see, e.g. $[4,5,7,8,15,16]$ ). By using the asymptotic behavior of elements in $\Phi(\tau)$, the further classification of the limit point case into the strong limit point case and the weak limit point case for high-order scalar differential equations was given by Everitt et al. in [17-19] and further studied in $[14,20]$. It was generalized to Hamiltonian differential systems by Qi and Chen [21] and well studied in [22]. For real valued functions $p(x)$ and $q(x)$, we say that (1.1) is in the strong limit point case at the end point $b$ if, for $y_{1}, y_{2} \in \Phi(\tau)$,

$$
p(x) y_{1}(x) y_{2}^{\prime}(x) \longrightarrow 0 \text { as } x \longrightarrow b
$$

In the present paper, we attempt to set up the analogues of the results (1.4) and (1.5) for (1.1) with complex valued coefficients $p$ and $q$. In the classification of Brown et al. in [12], Cases II and III depend on the admissible rotation angles (see Theorem 2.1). 
The exact dependence is set up in Theorem 2.5. We find that the asymptotic behavior of elements in $\Phi(\tau)$ also depends on the admissible rotation angles. So we first study the properties of the admissible angle set $E$ (defined in (2.10)) and prove that $E$ either contains a single point or is an interval. See Lemma 3.1. Then we introduce a pencil of Hamiltonian differential expressions with a new spectral parameter corresponding to (1.1) and set up the relationship between classifications of Hamiltonian differential expressions and (1.1). See Lemma 4.3. Applying the results mentioned in (1.4) and (1.5), we obtain sufficient and necessary conditions for Cases I and II involving weighted Sobolev spaces and the asymptotic behavior of elements in $\Phi(\tau)$. See Theorems 4.1 and 4.11. The main results of the present paper cover the result (1.4) (see Remark 4.2) and indicate that (1.4) means (1.5) when $E$ has more than one point; see Corollary 4.9.

Following this section, Section 2 gives some preliminary knowledge for (1.1) with complex valued coefficients, and Section 3 presents properties of the admissible rotation angle set $E$. The main results are given in Section 4 .

\section{Preliminary Knowledge}

Throughout this paper, we always assume that

(i) $p(x) \neq 0, w(x)>0$ a.e. on $[a, b)$ and $1 / p, q, w$ are all locally integrable on $[a, b)$,

(ii) $p$ and $q$ are complex valued, and

$$
\Omega=\overline{\mathrm{co}}\left\{\frac{q(x)}{w(x)}+r p(x): r>0, x \in[a, b)\right\} \neq \mathbb{C},
$$

where $\overline{\mathrm{co}}$ denotes the closed convex hull (i.e., the smallest closed convex set containing the exhibited set). Then, for each point on the boundary $\partial \Omega$, there exists a line through this point such that every point of $\Omega$ either lies in the same side of this line or is on it. That is, there exists a supporting line through this point. Let $K$ be a point on $\partial \Omega$. Denote by $L$ an arbitrary supporting line touching $\Omega$ at $K$, which may be the tangent to $\Omega$ at $K$ if it exists. We then perform a transformation of the complex plane $z \mapsto z-K$ and a rotation through an appropriate angle $\theta$ so that the image of $L$ coincides with the imaginary axis now and the set $\Omega$ is contained in the new right nonnegative half-plane.

For this purpose we introduce the set $S$ defined by

$$
S=\left\{(\theta, K): K \notin \Omega^{\circ}, \operatorname{Re}\left\{e^{i \theta}(\mu-K)\right\} \geq 0 \forall \mu \in \Omega\right\},
$$

where $\Omega^{\circ}$ is the interior of $\Omega$, and define the corresponding half-plane

$$
\Lambda_{\theta, K}=\left\{\mu \in \mathbb{C}: \operatorname{Re}\left\{e^{i \theta}(\mu-K)\right\}<0\right\} .
$$

Then, $\Lambda_{\theta, K} \subset \mathbb{C} \backslash \Omega$. From the definition of $S$, for all $x \in[a, b)$ and $0<r<\infty$,

$$
\operatorname{Re}\left\{e^{i \theta}\left[\frac{q(x)}{w(x)}+r p(x)-K\right]\right\} \geq 0
$$


The definition of $S$ is different from the corresponding one given by Brown et al. [12], but they are equivalent in describing square integrable solutions.

Besides, for $(\theta, K) \in S$

$$
\operatorname{Re}\left\{e^{i \theta}(\mu-K)\right\} \geq 0 \Longleftrightarrow \cos (\theta+\gamma) \geq 0 \quad \text { where } \mu-K=|\mu-K| e^{i \gamma}
$$

Using a nesting circle method based on that of both Weyl [1] and Sims, Brown et al. [12] divided (1.1) into three cases with respect to the corresponding half-planes $\Lambda_{\theta, K}$ as follows. The uniqueness referred to in the theorem and the following sections is only up to constant multiple.

Theorem 2.1 (cf. [12, Theorem 2.1]). Given a $(\theta, K) \in S$, the following three distinct cases are possible.

Case I. For all $\lambda \in \Lambda_{\theta, K}$, equation (1.1) has unique solution $y$ satisfying

$$
\int_{a}^{b}\left[\operatorname{Re}\left\{e^{i \theta} p\right\}\left|y^{\prime}\right|^{2}+\operatorname{Re}\left\{e^{i \theta}(q-K w)\right\}|y|^{2}\right]+\int_{a}^{b} w|y|^{2}<\infty
$$

and this is the only solution satisfying $y \in L_{w}^{2}$.

Case II. For all $\lambda \in \Lambda_{\theta, K}$, all solutions of (1.1) belong to $L_{w}^{2}$, and there exists unique solution of (1.1) satisfying (2.6).

Case III. For all $\lambda \in \Lambda_{\theta, K}$, all solutions of (1.1) satisfy (2.6).

Since every $\Lambda_{\theta, K}$ is a half-plane, it holds that

$$
\Lambda_{\theta_{1}, K_{1}} \bigcap \Lambda_{\theta_{2}, K_{2}} \neq \emptyset
$$

for $\left(\theta_{j}, K_{j}\right) \in S, j=1,2$, with $\theta_{1} \neq \theta_{2}(\bmod \pi)$. Note that $(2.4)$ implies that, for $0<r<\infty$ and $x \in[a, b)$,

$$
\operatorname{Re}\left\{e^{i \theta}\left(\frac{q(x)}{r w(x)}+p(x)-\frac{K}{r}\right)\right\} \geq 0
$$

Letting $r \rightarrow 0$ and $r \rightarrow \infty$ in (2.4) and (2.8), respectively, we have the following.

Lemma 2.2. For every $(\theta, K) \in S$ and $\lambda \in \Lambda_{\theta, K}$, there exists $\delta_{\lambda}(\theta)>0$ such that

$$
\operatorname{Re}\left\{e^{i \theta}(q-K w)\right\} \geq 0, \quad \operatorname{Re}\left\{e^{i \theta}(q-\lambda w)\right\} \geq \delta_{\lambda}(\theta) w, \quad \operatorname{Re}\left\{e^{i \theta} p\right\} \geq 0
$$

on $[a, b)$.

Using variation of parameters method, we can verify that, if all solutions of (1.1) belong to $L_{w}^{2}$ for some $\lambda_{0} \in \mathbb{C}$, then it is true for all $\lambda \in \mathbb{C}$. This also means the following. 
Lemma 2.3. If there exists $a\left(\theta_{0}, K_{0}\right) \in S$ such that (1.1) is in Case I with respect to (with respect to for short) $\Lambda_{\theta_{0}, K_{0}}$, then (1.1) is in Case I with respect to $\Lambda_{\theta, K}$ for every $(\theta, K) \in S$.

This indicates that Case I is independent of the choice of $(\theta, K) \in S$. But Cases II and III depend on the choice of $(\theta, K) \in S$ in general, that is, there may exist $\left(\theta_{1}, K_{1}\right),\left(\theta_{2}, K_{2}\right) \in S$ such that (1.1) is in Case II with respect to $\Lambda_{\theta_{1}, K_{1}}$ and is in Case III with respect to $\Lambda_{\theta_{2}, K_{2}}$. In order to make clear the dependence, we introduce the admissible angle set $E$ defined by

$$
E=\left\{\theta: \exists K \notin \Omega^{\circ},(\theta, K) \in S\right\} .
$$

Remark 2.4. For given $\theta \in E$, there exist many $K$ such that $(\theta, K) \in S$. In fact, if $\theta_{0} \in E$ with $\left(\theta_{0}, K_{0}\right) \in S$ for some $K_{0} \notin \Omega^{\circ}$, then for all $K \in L_{0},\left(\theta_{0}, K\right) \in S$

$$
L_{0}=\left\{\lambda \in \mathbb{C}: \operatorname{Re}\left\{e^{i \theta_{0}}\left(\lambda-K_{0}\right)\right\}=0\right\}
$$

The exact dependence of Cases II and III on $(\theta, K)$ can be given with the similar proof in [23, Theorem 2.1].

Theorem 2.5 (cf. [23, Theorem 2.1]). If there exists a $\left(\theta_{0}, K_{0}\right) \in S$ such that (1.1) is in Case II with respect to $\Lambda_{\theta_{0}, K_{0}}$, then (1.1) is in Case II with respect to $\Lambda_{\theta, K}$ for all $(\theta, K) \in S$ except for at most one $\theta_{1} \in E(\bmod \pi)$ such that $(1.1)$ is in Case III with respect to $\Lambda_{\theta_{1}, K_{1}}$.

Remark 2.6. Theorem 2.5 means that, if there exist $\theta_{j} \in E, j=1,2$, such that $\theta_{1} \neq \theta_{2}(\bmod \pi)$ and (1.1) is in Case III with respect to $\Lambda_{\theta_{j}, K_{j}}$ for $j=1,2$, then (1.1) is in Case III with respect to $\Lambda_{\theta, K}$ for all $(\theta, K) \in S$.

\section{Properties of the Angel Set $E$}

This section gives some properties of the set $E$, which will be used in the proof of our main results in Section 4 . In what follows, we say that $E$ has more than one point if there exist $\theta_{1}, \theta_{2} \in E$ with $\theta_{1} \neq \theta_{2}(\bmod \pi)$.

Lemma 3.1. Let $E$ be defined as in (2.10).

(i) The set $E$ is connected in the sense of $\bmod 2 \pi$.

(ii) If $E$ has more than one point, then, for every $\lambda \in \mathbb{C} \backslash \Omega$, there exist $\theta_{1}, \theta_{2} \in E$ with $\theta_{1}<\theta_{2}$ such that $\lambda \in \Lambda_{\theta, K}$ for $\theta \in\left(\theta_{1}, \theta_{2}\right) \subset E$.

Proof. (i) Suppose that $E$ has more than one point. Let $\theta_{1}, \theta_{2} \in E$ with $\theta_{1} \neq \theta_{2}(\bmod \pi)$; then $0<\theta_{2}-\theta_{1}<\pi(\bmod 2 \pi)$ or $\pi<\theta_{2}-\theta_{1}<2 \pi(\bmod 2 \pi)$.

If $0<\theta_{2}-\theta_{1}<\pi(\bmod 2 \pi)$ and $\left(\theta_{j}, K_{j}\right) \in S, j=1,2$, then we claim that $\left[\theta_{1}, \theta_{2}\right] \subset E$ $(\bmod 2 \pi)$. Let $L_{j}$ be the line similarly defined as $L_{0}$ with $K_{0}$ and $\theta_{0}$ replaced by $K_{j}$ and $\theta_{j}$, $j=1$, 2 . That is,

$$
L_{j}=\left\{\lambda \in \mathbb{C}: \operatorname{Re}\left\{e^{i \theta_{j}}\left(\lambda-K_{j}\right)\right\}=0\right\}, \quad K_{j} \notin \Omega^{\circ}, j=1,2
$$




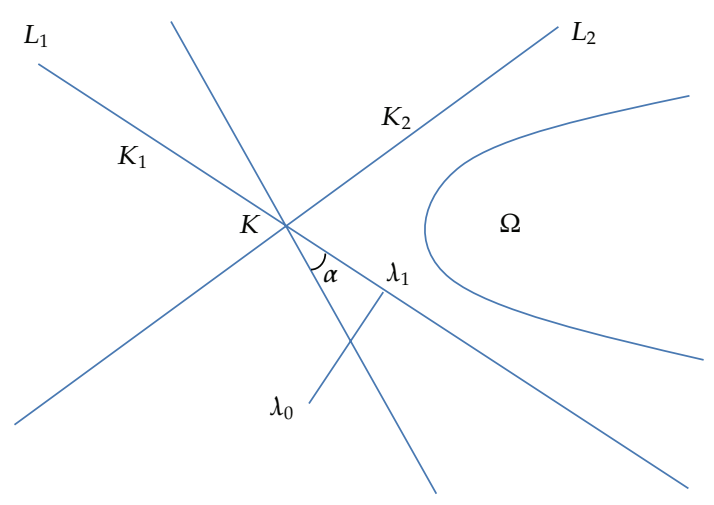

Figure 1: Figure of Lemma 3.1(ii).

Let $K$ be the intersection point of $L_{1}$ and $L_{2}$. Set

$$
\mu-K=|\mu-K| e^{i \gamma(\mu, K)}, \quad \mu \in \Omega .
$$

It follows from (2.5) that

$$
\cos \left(\gamma(\mu, K)+\theta_{j}\right) \geq 0, \quad \mu \in \Omega, j=1,2
$$

By $0<\theta_{2}-\theta_{1}<\pi(\bmod 2 \pi)$ and (3.3), we can get $\cos (\gamma(\mu, K)+\theta) \geq 0$ for $\theta \in\left[\theta_{1}, \theta_{2}\right]$ $(\bmod 2 \pi)$ on $\Omega$, which means $(\theta, K) \in S$ and $\theta \in E$.

According to the similar method, we can verify that, if $\pi<\theta_{2}-\theta_{1}<2 \pi(\bmod 2 \pi)$ and $\left(\theta_{j}, K_{j}\right) \in S, j=1,2$, then $\left[0, \theta_{1}\right] \cup\left[\theta_{2}, 2 \pi\right] \subset E(\bmod 2 \pi)$, that is, $\left[\theta_{2}, \theta_{1}\right] \subset E(\bmod 2 \pi)$.

(ii) For $\lambda_{0} \in \mathbb{C} \backslash \Omega$, choose $\left(\theta_{1}, K_{1}\right) \in S$ and $\delta_{0}>0$ such that $\lambda_{0} \in \Lambda_{\theta_{1}, K_{1}}$ and

$$
\operatorname{Re}\left\{e^{i \theta_{1}}\left(K_{1}-\lambda_{0}\right)\right\}=\delta_{0}>0 .
$$

Since $E$ has more than one point, we can choose $\theta_{2} \in E$ with $\theta_{2} \neq \theta_{1}(\bmod \pi)$. Without loss of generality, we suppose that $0<\theta_{2}-\theta_{1}<\pi(\bmod 2 \pi)$. Let $K$ be defined as in the proof of (i).

If $\lambda_{0} \in \Lambda_{\theta_{1}, K} \cap \Lambda_{\theta_{2}, K}$, then it follows from (2.3) that

$$
\cos \left(\gamma+\theta_{j}\right)<0, \quad j=1,2, \quad \text { where } \lambda_{0}-K=\left|\lambda_{0}-K\right| e^{i \gamma}
$$

By $0<\theta_{2}-\theta_{1}<\pi(\bmod 2 \pi)$ and (3.5), we can get $\cos (\gamma+\theta)<0$ for $\theta \in\left[\theta_{1}, \theta_{2}\right](\bmod 2 \pi)$, which means $\lambda_{0} \in \Lambda_{\theta, K}$ for $\theta \in\left[\theta_{1}, \theta_{2}\right]$.

Suppose that $\lambda_{0} \notin \Lambda_{\theta_{2}, K}$. Let $\lambda_{1} \in L_{1}$ be the unique point such that $\delta_{0}=\operatorname{dist}\left(\lambda_{0}, L_{1}\right)=$ $\operatorname{dist}\left(\lambda_{1}, \lambda_{0}\right)$. Let $\alpha=\arctan \left|\lambda_{0}-\lambda_{1}\right| / 2\left|K-\lambda_{1}\right|$; then $\alpha+\theta_{1} \in\left(\theta_{1}, \theta_{2}\right) \subset E$ by $\lambda_{0} \notin \Lambda_{\theta_{2}, K}$, and $\lambda_{0} \in \Lambda_{\alpha+\theta_{1}, K}$ by the definition of $\alpha$ (see Figure 1 ).

So, we can get that $\lambda_{0} \in \Lambda_{\theta, K}$ for $\theta \in\left[\theta_{1}, \theta_{1}+\alpha\right]$ by $\lambda_{0} \in \Lambda_{\theta_{1}, K} \cap \Lambda_{\theta_{1}+\alpha, K}$, and the lemma is proved. 


\section{Asymptotic Behavior}

In this section, we will give asymptotic behavior of elements in the natural domain of the formally differential operator $\tau$ defined on the interval $[0, \infty)$ with 0 being a regular end point and $+\infty$ being implicitly a singular end point. All results in this section can be stated for any singular end point, left or right on an arbitrary interval $(a, b)$, where $-\infty \leq a<b \leq+\infty$. Recall that $(1.1)$ on $(a, b)$ is said to be regular at $a$ if $1 / p, q$ and $w$ are integrable on $(a, c)$ for some (and hence any) $c \in(a, b)$ and singular at $a$ otherwise; the regularity and singularity at $b$ are defined similarly (cf. [24]). Note that the regularity (resp., singularity) of an end point is solely determined by the integrability (resp., nonintegrability) of the coefficients in (1.1) at the end point, not the finiteness (resp., infiniteness) of the end point, as already remarked by Atkinson at the end of [13, Section 9.1]. See also [10, Theorem 2.3.1]. Recall the definition of $\Phi(\tau)$ in (1.3). We also define

$$
\Phi(\bar{\tau})=\left\{y \in L_{w}^{2}: y, \bar{p} y^{\prime} \in \mathrm{AC}_{\mathrm{loc}}, \bar{\tau} y \in L_{w}^{2}\right\}
$$

where

$$
\bar{\tau} y:=w^{-1}\left[-\left(\bar{p} y^{\prime}\right)^{\prime}+\bar{q} y\right] \quad \text { on }[0, \infty)
$$

The first result of this section is as follows.

Theorem 4.1. (i) $\tau$ is in Case I if and only if for $y_{1}, y_{2} \in \Phi(\tau)$ and $\theta \in E$

$$
\overline{p(x)} y_{2}(x) \overline{y_{1}^{\prime}(x)}+e^{2 i \theta} p(x) \overline{y_{1}(x)} y_{2}^{\prime}(x) \longrightarrow 0 \quad \text { as } x \longrightarrow \infty
$$

(ii) $\tau$ is in Case I if and only if for $y_{1} \in \Phi(\tau), y_{2} \in \Phi(\bar{\tau})$

$$
p(x)\left[y_{2}(x) \overline{y_{1}^{\prime}(x)}-\overline{y_{1}(x)} y_{2}^{\prime}(x)\right] \longrightarrow 0 \quad \text { as } x \longrightarrow \infty
$$

Remark 4.2. Clearly $\Phi(\bar{\tau})=\overline{\Phi(\tau)}$, by the definition of $\boldsymbol{\Phi}(\bar{\tau})$. It is easy to see that (4.4) is equivalent to

$$
p(x)\left[y_{2}(x) y_{1}^{\prime}(x)-y_{1}(x) y_{2}^{\prime}(x)\right] \longrightarrow 0 \quad \text { as } x \longrightarrow \infty
$$

for $y_{1}, y_{2} \in \Phi(\tau)$.

We will use spectral theory of Hamiltonian differential systems to prove Theorem 4.1, so that we first prepare some known results for the Hamiltonian differential system

$$
\begin{aligned}
u^{\prime} & =A u+B v+\xi W_{2} v, \\
v^{\prime} & =C u-A^{*} v-\xi W_{1} u, \quad \text { on }[0, \infty)
\end{aligned}
$$


where $u, v$ are $\mathbb{C}^{n}$ valued functions, $u^{T}$ is the transpose of $u, A, B, C, W_{1}$, and $W_{2}$ are locally integrable, complex valued $n \times n$ matrices on $[0, \infty), B, C, W_{1}$, and $W_{2}$ are Hermit matrices and $W_{1}(t)>0, W_{2}(t) \geq 0$ on $[0, \infty)$, and $\xi$ is the spectral parameter. Assume that the definiteness condition (see, e.g., [13, Chapter 9, page 253]) holds:

$$
\int_{0}^{\infty} y^{*} W y>0 \text { for each nontrivial solution } y \text { of (4.6), }
$$

where $W=\operatorname{diag}\left(W_{1}, W_{2}\right)$. Let $L_{W}^{2}:=L_{W}^{2}[0, \infty)$ denote the space of Lebesgue measurable $2 n$ dimensional functions $f$ satisfying $\int_{0}^{\infty} f^{*}(s) W(s) f(s) d s<\infty$. We say that $(4.6)$ is in the limit point case at infinity if there exists exactly $n^{\prime}$ s solutions of (4.6) belonging to $L_{W}^{2}$ for $\xi \in \mathbb{C}$ with $\operatorname{Im} \xi \neq 0$.

Let $\Phi$ be the maximal domain associated with (4.6), that is, $\left(u^{T}, v^{T}\right)^{T} \in \Phi$ if and only if $\left(u^{T}, v^{T}\right)^{T} \in \mathrm{AC}_{\mathrm{loc}} \cap L_{W}^{2}$, and there exists an element $\left(f^{T}, g^{T}\right)^{T} \in L_{W}^{2}$ such that

$$
\begin{aligned}
u^{\prime} & =A u+B v+\xi W_{2} v+W_{2} g \\
v^{\prime} & =C u-A^{*} v-\xi W_{1} u-W_{1} f, \quad \text { on }[0, \infty)
\end{aligned}
$$

It is well known (cf. $[5,7])$ that $(4.6)$ is in the limit point case at infinity if and only if

$$
Y_{1}^{*}(x) J Y_{2}(x) \longrightarrow 0 \text { as } x \longrightarrow \infty, J=\left(\begin{array}{cc}
0 & -I_{n} \\
I_{n} & 0
\end{array}\right)
$$

for $Y_{1}, Y_{2} \in \Phi$, and for every $\xi \in \mathbb{C}$ with $\operatorname{Im} \xi \neq 0$ there exists a Green function $G(t, s, \xi)$ such that, for $F=\left(f^{T}, g^{T}\right)^{T} \in L_{W}^{2}$,

$$
Y=\left(\begin{array}{l}
u \\
v
\end{array}\right)=T_{\xi} F \in L_{W}^{2}, \quad \text { satisfies (4.8), }
$$

where $\left(T_{\xi} F\right)(x)=\int_{0}^{\infty} G(x, s, \xi) W(s) F(s) \mathrm{d} s$.

Let $(\theta, K) \in S$ and choose $\lambda_{0} \in \Lambda_{\theta, K}$. Then from (2.9), one sees that

$$
\operatorname{Re}\left\{e^{i \theta}\left(q-\lambda_{0} \omega\right)\right\} \geq \delta_{0} \omega>0, \quad \operatorname{Re}\left\{e^{i \theta}\left(q-K_{0} w\right)\right\} \geq 0, \quad \operatorname{Re}\left\{e^{i \theta} p\right\} \geq 0
$$

for some $\delta_{0}>0$. Set

$$
\begin{array}{cl}
r_{1}(x)=\left|q(x)-\lambda_{0} w(x)\right|, \quad & q(x)-\lambda_{0} w(x)=r_{1}(x) e^{i \alpha(x)}, \quad \alpha_{1}(x)=\theta+\alpha(x), \\
r_{2}(x)=|p(x)|, \quad & p(x)=r_{2}(x) e^{i \beta(x)}, \quad \beta_{1}(x)=\theta+\beta(x) .
\end{array}
$$


Consider the Hamiltonian differential system (4.6) with $n=1, A(x) \equiv 0$ and

$$
\begin{gathered}
C(x)=r_{1}(x) \sin \alpha_{1}(x), \quad W_{1}(x):=w_{1}(x)=r_{1}(x) \cos \alpha_{1}(x), \\
B(x)=\frac{\sin \beta_{1}(x)}{r_{2}(x)}, \quad W_{2}(x):=w_{2}(x)=\frac{\cos \beta_{1}(x)}{r_{2}(x)},
\end{gathered}
$$

that is, the 2-dimensional Hamiltonian differential system

$$
H(\theta): u^{\prime}=B v+\xi w_{2} v, \quad v^{\prime}=C u-\xi w_{1} u .
$$

It follows from (4.11) that

$$
w_{1}=\operatorname{Re}\left\{e^{i \theta}\left(q-\lambda_{0} w\right)\right\} \geq \delta_{0} w>0, \quad w_{2}=\frac{\operatorname{Re}\left\{e^{i \theta} p(t)\right\}}{r_{2}^{2}} \geq 0,
$$

and it is easy to verify that the definiteness condition holds for the system (4.14). In fact, $y$ is a solution of (1.1) if and only if $(u, v)^{T}$ is a solution of (4.14) with

$$
u=y, \quad v=-i e^{i \theta} p y^{\prime} .
$$

This fact immediately yields the following result which is frequently used in the proof of Theorems 4.1 and 4.11 .

Lemma 4.3. (i) $\tau$ is in Case I or Case II with respect to $(\theta, K) \in S$ if and only if $H(\theta)$ is in the limit point case at $\infty$.

(ii) $\tau$ is in Case III with respect to $(\theta, K) \in S$ if and only if $H(\theta)$ is in the limit circle case at $\infty$.

Lemma 4.4. If $E$ has more than one point, then $\Phi_{\theta}(\tau) \equiv \Phi_{S}(\tau)$ on $E^{o}$, the interior of $E$, where

$$
\begin{gathered}
\Phi_{\theta}(\tau)=\left\{y \in \Phi(\tau): \int_{0}^{\infty}\left[\operatorname{Re}\left\{e^{i \theta} p\right\}\left|y^{\prime}\right|^{2}+\operatorname{Re}\left\{e^{i \theta} q\right\}|y|^{2}\right]<\infty\right\}, \\
\Phi_{S}(\tau)=\left\{y \in \Phi(\tau): \int_{0}^{\infty}\left[|p|\left|y^{\prime}\right|^{2}+|q||y|^{2}\right]<\infty\right\} .
\end{gathered}
$$

Proof. Let $\theta_{1} \in E^{o}$ be fixed. There exist $\theta_{2}, \theta_{3} \in E^{o}$ such that

$$
\theta_{3}<\theta_{1}<\theta_{2} \bmod (2 \pi), \quad 0<\theta_{2}-\theta_{3}<\frac{\pi}{2 \bmod (2 \pi)}, \quad \bigcap_{j=1}^{3} \Lambda_{\theta_{j}, K_{j}} \neq \emptyset
$$

by Lemma 3.1. Choose $\lambda_{0} \in \bigcap_{j=1}^{3} \Lambda_{\theta_{j}, K_{j}}$. Letting $\beta:=\beta(x)$ be defined as in (4.12) and solving $\cos \left(\theta_{1}+\beta\right)$ from the equations

$$
\cos \left(\theta_{j}+\beta\right)=\cos \left(\theta_{1}+\beta\right) \cos \left(\theta_{j}-\theta_{1}\right)-\sin \left(\theta_{1}+\beta\right) \sin \left(\theta_{j}-\theta_{1}\right), \quad j=2,3,
$$


we have that $\cos \left(\theta_{1}+\beta\right)=C_{1} \cos \left(\theta_{2}+\beta\right)+C_{2} \cos \left(\theta_{3}+\beta\right)$ with

$$
C_{1}=\frac{\sin \left(\theta_{1}-\theta_{3}\right)}{\sin \left(\theta_{2}-\theta_{3}\right)}>0, \quad C_{2}=\frac{\sin \left(\theta_{2}-\theta_{1}\right)}{\sin \left(\theta_{2}-\theta_{3}\right)}>0
$$

by (4.18). Since $\int_{0}^{\infty} \operatorname{Re}\left\{e^{i \theta_{1}} p\right\}\left|y^{\prime}\right|^{2}<\infty$ for $y \in \Phi_{\theta_{1}}(\tau)$, we have that

$$
C_{1} \int_{0}^{\infty} \operatorname{Re}\left\{e^{i \theta_{2}} p\right\}\left|y^{\prime}\right|^{2}+C_{2} \int_{0}^{\infty} \operatorname{Re}\left\{e^{i \theta_{3}} p\right\}\left|y^{\prime}\right|^{2}=\int_{0}^{\infty} \operatorname{Re}\left\{e^{i \theta_{1}} p\right\}\left|y^{\prime}\right|^{2}<\infty,
$$

and hence $\int_{0}^{\infty} \operatorname{Re}\left\{e^{i \theta_{2}} p\right\}\left|y^{\prime}\right|^{2}<\infty$ for $y \in \Phi_{\theta_{1}}(\tau)$. The same proof as the above with $\beta$ replaced by $\alpha$ also proves $\int_{0}^{\infty} \operatorname{Re}\left\{e^{i \theta_{2}}\left(q-\lambda_{0} w\right)\right\}|y|^{2}<\infty$ for $y \in \Phi_{\theta_{1}}(\tau)$, where $\alpha:=\alpha(x)$ is defined as in (4.12). Therefore, for $y \in \Phi_{\theta_{1}}(\tau)$,

$$
\int_{0}^{\infty}\left[\operatorname{Re}\left\{e^{i \theta_{j}} p\right\}\left|y^{\prime}\right|^{2}\right], \quad \int_{0}^{\infty} \operatorname{Re}\left\{e^{i \theta_{j}}\left(q-\lambda_{0} w\right)\right\}|y|^{2}<\infty, \quad j=1,2
$$

Set $p_{\theta}=e^{i \theta} p$ and $q_{\theta}=e^{i \theta}\left(q-\lambda_{0} w\right)$. It follows from

$$
\sin ^{2}\left(\theta_{2}-\theta_{1}\right)=\cos ^{2} \theta_{2}+\cos ^{2} \theta_{1}-2 \cos \theta_{2} \cos \theta_{1} \cos \left(\theta_{1}-\theta_{2}\right) \leq\left(\cos \theta_{2}+\cos \theta_{1}\right)^{2}
$$

and (4.15) that

$$
\operatorname{Re}\left(p_{\theta_{1}}+p_{\theta_{2}}\right) \geq \varepsilon_{0}|p|, \quad \operatorname{Re}\left(q_{\theta_{1}}+q_{\theta_{2}}\right) \geq \varepsilon_{0}|q-\lambda w|, \quad \varepsilon_{0}=\sin \left(\theta_{2}-\theta_{1}\right) .
$$

Then (4.24) and (4.22) yield that, for $y \in \Phi_{\theta_{1}}(\tau)$,

$$
\int_{0}^{\infty}|p|\left|y^{\prime}\right|^{2}, \quad \int_{0}^{\infty}\left|q-\lambda_{0} w\right||y|^{2}<\infty
$$

Note that $y \in L_{w}^{2}$. Then (4.25) gives $y \in \Phi_{S}(\tau)$, or $\Phi_{\theta_{1}}(\tau) \subset \Phi_{S}(\tau)$. Clearly, $\Phi_{S}(\tau) \subset \Phi_{\theta_{1}}(\tau)$. Thus $\Phi_{\theta_{1}}(\tau)=\Phi_{S}(\tau)$.

Lemma 4.4 indicates the following.

Corollary 4.5. If $\tau$ is in Case II with respect to some $\left(\theta_{0}, K_{0}\right) \in S$ and $E$ has more than one point, then Case III only occurs at the end point of E.

Proof. If $\tau$ is in Case III with respect to some $\left(\theta_{1}, K_{1}\right) \in S$ with $\theta_{1} \in E^{o}$, then $\boldsymbol{\Phi}(\tau)=\Phi_{\theta_{1}}(\tau)$ is restricted in the solution space of (1.1) by the definition of Case III. Since $\Phi_{\theta_{1}}(\tau)=\Phi_{S}(\tau)$ by Lemma 4.4, we have that $\Phi(\tau)=\Phi_{S}(\tau)$ restricted in the solution space of (1.1). This means that all solutions of (1.1) with $\lambda \in \Lambda_{\theta_{1}, K_{1}}$ satisfy

$$
\int_{0}^{\infty}\left(|p|\left|y^{\prime}\right|^{2}+|q||y|^{2}\right)<\infty
$$


Using variation of parameters method we can prove that it is true for all $\lambda \in \mathbb{C}$, and hence $\tau$ is in Case III with respect to $\left(\theta_{0}, K_{0}\right)$, a contradiction.

Lemma 4.6. If $\tau$ is in Case I and $y \in \Phi(\tau)$, then $(y, v)^{T} \in \Phi(\theta)$ with $v=-i e^{i \theta} p y^{\prime}$, where $\Phi(\theta)$ is the maximal domain associated with (4.14).

Proof. Suppose that $\tau$ is in Case I with respect to $(\theta, K) \in S$. We claim that $\boldsymbol{\Phi}(\tau)=\Phi_{\theta}(\tau)$. Set

$$
\left(\tau-\lambda_{0}\right) y_{0}=w^{-1}\left[-\left(p y_{0}^{\prime}\right)^{\prime}+\left(q-\lambda_{0} w\right) y_{0}\right]=g_{0}
$$

for $y_{0} \in \Phi(\tau)$ and $\lambda_{0} \in \Lambda_{\theta, K}$.

Set $u_{0}=y_{0}, v_{0}=-i e^{i \theta} p y_{0}^{\prime}$. Then $\left(u_{0}, v_{0}\right)$ satisfies

$$
u^{\prime}=B v+i w_{2} v, \quad v^{\prime}=C u-i w_{1} u-w_{1} f_{1}, \quad f_{1}=\frac{w}{w_{1}}\left(-i e^{i \theta} g_{0}\right) .
$$

Conversely, if $(u, v)$ satisfies (4.28), then $y=u$ solves (4.27). Note that $g_{0} \in L_{w}^{2}$, or $-i e^{i \theta_{0}} g_{0} \in$ $L_{w}^{2}$, and $w_{1} \geq \delta w$ implies $f_{1} \in L_{w_{1}}^{2}$.

Considering (4.28), we get from (4.10) that (4.28) has a solution $\left(u_{1}, v_{1}\right)^{T}$ such that $u_{1} \in L_{w_{1}}^{2}, v_{1} \in L_{w_{2}}^{2}$ and $v_{1}=-i e^{i \theta_{0}} p u_{1}^{\prime}$. Set $y_{1}=u_{1}$. Then $y_{1}$ satisfies (4.27), and hence $(\tau-$ $\left.\lambda_{0}\right)\left(y_{0}-y_{1}\right)=0$. Note that $y_{1}=u_{1} \in L_{w_{1}}^{2}$, and $w_{1} \geq \delta w$ implies that $y_{1} \in L_{w}^{2}$. Thus, $y_{1}-y_{0}$ is an $L_{w}^{2}$-solution of $\tau y=\lambda_{0} y$. Since $\tau$ is in Case I with respect to $\left(\theta_{0}, K_{0}\right)$, it follows from (2.6) that $y_{1}-y_{0} \in L_{w_{1}}^{2}$ and $v_{1}-v_{0} \in L_{w_{2}}^{2}$. This together with $y_{1} \in L_{w_{1}}^{2}$ and $v_{1} \in L_{w_{2}}^{2}$ gives $y_{0} \in L_{w_{1}}^{2}$ and $v_{0} \in L_{w_{2}}^{2}$. In fact, we have proved that, for $y \in \Phi(\tau)$,

$$
\int_{0}^{\infty}\left|q-\lambda_{0} w\right| \cos \alpha_{1}|y|^{2}<\infty, \quad \int_{0}^{\infty}|p| \cos \beta_{1}\left|y^{\prime}\right|^{2}<\infty
$$

or

$$
\int_{0}^{\infty}\left[\operatorname{Re}\left\{e^{i \theta} p\right\}\left|y^{\prime}\right|^{2}+\operatorname{Re}\left\{e^{i \theta}\left(q-\lambda_{0} w\right)\right\}|y|^{2}\right]<\infty
$$

where $\alpha_{1}$ and $\beta_{1}$ are defined in (4.12) or (4.13). Since $y \in L_{w}^{2},(4.30)$ means that

$$
\int_{0}^{\infty}\left[\operatorname{Re}\left\{e^{i \theta} p\right\}\left|y^{\prime}\right|^{2}+\operatorname{Re}\left\{e^{i \theta} q\right\}|y|^{2}\right]<\infty
$$

or $y \in \Phi_{\theta}(\tau)$, and hence $\Phi(\tau)=\Phi_{\theta}(\tau)$. Recall that $f_{1} \in L_{w_{1}}^{2}$. Then (4.30) and (4.28) imply that, if $y \in \Phi(\tau)$, then $(y, v)^{T} \in \Phi(\theta)$.

Corollary 4.7. If $\tau$ is in Case I and $y \in \Phi(\bar{\tau})$, then $(y, v)^{T} \in \Phi(\theta)$ with $v=i e^{-i \theta} \bar{p} y^{\prime}$.

Proof. For $y \in \Phi(\bar{\tau}), \bar{y} \in \Phi(\tau)$ by $\Phi(\bar{\tau})=\overline{\Phi(\tau)}$. So $(\bar{y}, \bar{v})^{T} \in \Phi(\theta)$ with $\bar{v}=-i e^{i \theta} p \bar{y}^{\prime}$ by Lemma 4.6. Clearly $\boldsymbol{\Phi}(\theta)=\overline{\boldsymbol{\Phi}(\theta)}$ since $H(\theta)$ is symmetrical. Then we have that $(y, v)^{T} \in \mathscr{\Phi}(\theta)$ with $v=i e^{-i \theta} \bar{p} y^{\prime}$. 
Proof of Theorem 4.1. The proof of (i): suppose that $\tau$ is in Case I. Since (4.14) is in the limit point case at infinity by Lemma 4.3 , we know that (4.9) holds for all $\Upsilon_{1}, Y_{2} \in \Phi(\theta)$. For $y_{1}, y_{2} \in$ $\Phi(\tau)$, since $\left(y_{j}, v_{j}\right)^{T} \in \Phi(\theta)$ with $v_{j}=-i e^{i \theta} p y_{j}^{\prime}, j=1,2$, by Lemma 4.6, it follows from (4.9) that

$$
\left(\bar{y}_{1}, \bar{v}_{1}\right)\left(\begin{array}{cc}
0 & -1 \\
1 & 0
\end{array}\right)\left(\begin{array}{l}
y_{2} \\
v_{2}
\end{array}\right)=i e^{-i \theta}\left(\overline{p y}_{1}^{\prime} y_{2}+e^{2 i \theta} p \bar{y}_{1} y_{2}^{\prime}\right) \longrightarrow 0
$$

as $x \rightarrow \infty$.

Conversely, assume that (4.3) holds for all elements of $\Phi(\tau)$. We claim that (1.1) must be in Case I. Suppose on the contrary that (1.1) is not in Case I. Then all solutions of (1.1) belong to $L_{w}^{2}$ for $\lambda \in \mathbb{C}$. Choose $\lambda_{0} \in \Lambda_{\theta, K}$, and let $y_{0}$ be a nontrivial solution of (1.1) satisfying $y_{0}(0)=0$. Then $y_{0} \in \Phi(\tau)$ by $y_{0} \in L_{w}^{2}$. Furthermore, it follows from $\left(\tau-\lambda_{0}\right) y_{0}=0$ that

$$
-\left(p y_{0}^{\prime}\right)^{\prime} \bar{y}_{0}+\left(q-\lambda_{0} w\right)\left|y_{0}\right|^{2}=0, \quad-\left(\overline{p y}_{0}^{\prime}\right)^{\prime} y_{0}+\left(\bar{q}-\bar{\lambda}_{0} w\right)\left|y_{0}\right|^{2}=0
$$

Integrating (4.33) on $[0, x]$ we have that

$$
\begin{aligned}
& -\left.\left(p y_{0}^{\prime}\right) \bar{y}_{0}\right|_{0} ^{x}+\int_{0}^{x}\left[p\left|y_{0}^{\prime}\right|^{2}+\left(q-\lambda_{0} w\right)\left|y_{0}\right|^{2}\right]=0, \\
& -\left.\left(\overline{p y}_{0}^{\prime}\right) y_{0}\right|_{0} ^{x}+\int_{0}^{x}\left[\bar{p}\left|y_{0}^{\prime}\right|^{2}+\left(\bar{q}-\bar{\lambda}_{0} w\right)\left|y_{0}\right|^{2}\right]=0 .
\end{aligned}
$$

Multiplying $e^{i \theta}$ and $e^{-i \theta}$ to the first and second equalities in (4.34), respectively, and adding them together, we have that

$$
-\left[e^{i \theta}\left(p y_{0}^{\prime}\right) \bar{y}_{0}+e^{-i \theta}\left(\overline{p y}_{0}^{\prime}\right) y_{0}\right](x)+\int_{0}^{x}\left[\left(p_{\theta}+\bar{p}_{\theta}\right)\left|y_{0}^{\prime}\right|^{2}+\left(q_{\theta}+\bar{q}_{\theta}\right)\left|y_{0}\right|^{2}\right]=0
$$

since $y_{0}(0)=0$, where $p_{\theta}=e^{i \theta} p$ and $q_{\theta}=e^{i \theta}\left(q-\lambda_{0} w\right)$. Note that

$$
\left[e^{i \theta}\left(p y_{0}^{\prime}\right) \bar{y}_{0}+e^{-i \theta}\left(\overline{p y}_{0}^{\prime}\right) y_{0}\right](x)=e^{-i \theta}\left[\overline{p y}_{0}^{\prime} y_{0}+e^{2 i \theta} p y_{0}^{\prime} \bar{y}_{0}\right](x) \longrightarrow 0
$$

as $x \rightarrow \infty$ by assumption (4.3) and

$$
\operatorname{Re} p_{\theta}=\operatorname{Re}\left\{e^{i \theta} p\right\} \geq 0, \quad \operatorname{Re} q_{\theta}=\operatorname{Re}\left\{e^{i \theta}\left(q-\lambda_{0} w\right)\right\} \geq \delta_{0} w
$$

by (4.15). Then letting $x \rightarrow \infty$ in (4.35), we have a contradiction. This proves the first part of this theorem. 
The proof of (ii): suppose that $\tau$ is in Case I. Set $v_{1}=-i e^{i \theta} p y_{1}^{\prime}, v_{2}=i e^{-i \theta} \bar{p} y_{2}^{\prime}$ for $y_{1} \in$ $\boldsymbol{\Phi}(\tau), y_{2} \in \Phi(\bar{\tau})$. Then, we can get $\left(y_{1}, v_{1}\right)^{T} \in \Phi(\theta)$ by Lemma 4.6 and $\left(y_{2}, v_{2}\right)^{T} \in \Phi(\theta)$ by Corollary 4.7. Hence

$$
\left(\bar{y}_{1}, \bar{v}_{1}\right)\left(\begin{array}{cc}
0 & -1 \\
1 & 0
\end{array}\right)\left(\begin{array}{l}
y_{2} \\
v_{2}
\end{array}\right)=i e^{-i \theta}\left(\overline{p y}_{1}^{\prime} y_{2}-\overline{p y}_{1} y_{2}\right) \longrightarrow 0
$$

as $x \rightarrow \infty$ by (4.9), that is, $p(x)\left[y_{2}(x) \overline{y_{1}^{\prime}(x)}-\overline{y_{1}(x)} y_{2}^{\prime}(x)\right] \rightarrow 0$ as $x \rightarrow \infty$.

Conversely, if $\tau$ is not in Case I, then all solutions of (1.1) belong to $L_{w}^{2}$ for $\lambda \in \mathbb{C}$. Let $y_{i}, i=1,2$, be the solution of $\left(\tau-\lambda_{0}\right) y=0$ such that

$$
\left(\begin{array}{c}
p y_{1}^{\prime}(0) \\
y_{1}(0)
\end{array}\right)=\left(\begin{array}{l}
1 \\
0
\end{array}\right), \quad\left(\begin{array}{c}
p y_{2}^{\prime}(0) \\
y_{2}(0)
\end{array}\right)=\left(\begin{array}{l}
0 \\
1
\end{array}\right) \text {. }
$$

Since $y_{i} \in L_{w}^{2}, y_{i} \in D(\tau), i=1,2$. Then the Wronskian

$$
\left|\begin{array}{ll}
p y_{1}^{\prime} & y_{1} \\
p y_{2}^{\prime} & y_{2}
\end{array}\right|=p\left(y_{1}^{\prime} y_{2}-y_{1} y_{2}^{\prime}\right) \equiv 1
$$

which contradicts condition (4.5). See Remark 4.2.

Remark 4.8. If $q(x)$ and $p(x)$ are real valued, then $\Omega \subset \mathbb{R}$ and $(\theta, K)=( \pm \pi / 2,0) \in S$ with $\operatorname{Re}\left\{e^{i \theta} p(x)\right\}=\operatorname{Re}\left\{e^{i \theta}(q(x)-K w(x))\right\} \equiv 0$. This means that Case I, Cases II and III reduce to Weyl's limit point, limit-circle cases, respectively. For this case, we know that (1.1) is in the limit point case at $\infty$ if and only if

$$
p(x)\left[y_{2}(x) \overline{y_{1}^{\prime}}(x)-\overline{y_{1}}(x) y_{2}^{\prime}(x)\right] \longrightarrow 0 \quad \text { as } x \longrightarrow \infty
$$

for $y_{1}, y_{2} \in \Phi(\tau)$, that is, (1.4). Clearly, if $p$ is real valued and $\pi / 2 \in E$, then (4.3) reduces to (1.4). Therefore, (4.3) is a generalization of (1.4).

Corollary 4.9. If $E$ has more than one point, then $\tau$ is in Case I if and only if, for $y_{1}, y_{2} \in \boldsymbol{D}(\tau)$,

$$
p(x) y_{1}(x) y_{2}^{\prime}(x) \longrightarrow 0 \quad \text { as } x \longrightarrow \infty
$$

That is $\tau$ is in the strong limit point case at $\infty$.

Proof. Suppose that $E$ has more than one point and $\tau$ is in Case I. Choose $\theta_{j} \in E, j=1,2$, with $\theta_{1} \neq \theta_{2}(\bmod \pi)$. Then (4.3) holds for $\theta=\theta_{j}, j=1,2$. This gives that for $y_{1}, y_{2} \in \Phi(\tau)$

$$
\left(e^{2 i \theta_{1}}-e^{2 i \theta_{2}}\right) p \bar{y}_{1} y_{2}^{\prime} \longrightarrow 0 \quad \text { as } x \longrightarrow \infty,
$$

and hence $(4.42)$ holds since $\theta_{1} \neq \theta_{2}(\bmod \pi)$. 
Conversely, assume that (4.42) holds for all $y_{i} \in \Phi(\tau), i=1$, 2. Since (4.42) implies (4.3), we conclude from (i) of Theorem 4.1 that $\tau$ is in Case I.

Corollary 4.10. If $\tau$ is symmetric and $q(x) \geq q_{0} w(x)$ on $[0, \infty)$, then $\tau$ is in the limit point case at $\infty$ if and only if it is in the strong limit point case at $\infty$.

Proof. Note that for, $\theta \in[-\pi / 2, \pi / 2],\left(\theta, q_{0}\right) \in S$. Then $[-\pi / 2, \pi / 2] \in E$. Therefore, (4.3) holds if and only if (4.42) holds by Corollary 4.9.

Theorem 4.11. $\tau$ is in Case II with respect to $\left(\theta_{0}, K_{0}\right) \in S$ if and only if $\Phi(\tau) \neq \Phi_{\theta_{0}}(\tau) \neq \emptyset$ and (4.3) holds for $y_{1}, y_{2} \in \Phi_{\theta_{0}}(\tau)$.

Proof. Suppose that $\tau$ is in Case II with respect to some $(\theta, K) \in S$. By the definition of Case II we know that $\Phi_{\theta}(\tau)$ is nonempty and $\Phi(\tau) \neq \Phi_{\theta}(\tau)$. With a similar proof to that one in the first part of (i) in Theorem 4.1, we can get that (4.3) holds for $y_{1}, y_{2} \in \Phi_{\theta_{0}}(\tau)$ by Lemma 4.3 and (4.32).

Conversely, suppose that $\Phi(\tau) \neq \Phi_{\theta}(\tau)$ for some $(\theta, K) \in S$ and (4.3) holds for $y \in$ $\Phi_{\theta}(\tau)$. By the proof of Lemma 4.6, we know that $\tau$ is not in Case I with respect to $(\theta, K)$. We only need to prove that $\tau$ is not in Case III with respect to this $(\theta, K)$. If it is not true, then all solutions of (1.1) with $\lambda \in \Lambda_{\theta, K}$ satisfy (2.6) and so belong to $\Phi_{\theta}(\tau)$. Let $y_{0}$ be a nontrivial solution of (1.1) with $y(0)=0$. Then $y_{0} \in \Phi_{\theta}(\tau)$, and hence the same proof as in (4.33)-(4.35) yields a contradiction.

Corollary 4.12. If $E$ has more than one point, then $\tau$ is in case II with respect to some $(\theta, K) \in S$ if and only if $\Phi(\tau) \neq \Phi_{S}(\tau)$ and (4.42) holds for $y_{1}, y_{2} \in \Phi_{\theta}(\tau)$ with $\theta \in E^{o}$.

Proof. If $E$ has more than one point and $\tau$ is in Case II with respect to some $\left(\theta_{0}, K_{0}\right) \in S$, then there exists $\theta_{1} \in E^{o}$ such that $\tau$ is in Case II with respect to $\left(\theta_{1}, K_{1}\right) \in S$ by Theorem 2.5. Since $\Phi_{\theta_{1}}(\tau) \subsetneq \Phi(\tau)$ by Theorem 4.11 and $\Phi_{S}(\tau)=\Phi_{\theta_{1}}(\tau)$ by Lemma 4.4, one sees that $\Phi(\tau) \neq \Phi_{S}(\tau)$.

Choose $\theta_{2} \in E^{o}$ with $\theta_{1} \neq \theta_{2}(\bmod \pi)$ such that $\tau$ is in Case II with respect to $\left(\theta_{j}, K_{j}\right) \in$ $S$ for $j=1,2$ by Theorem 2.5. Then (4.3) holds for $\theta=\theta_{j}, j=1,2$ by Theorem 4.11. Since $\Phi_{\theta_{j}}(\tau)=\Phi_{S}(\tau)$, the same proof as in (4.43) gives that (4.42) holds for $y_{1}, y_{2} \in \Phi_{S}(\tau)$.

Conversely, suppose that $\boldsymbol{\Phi}(\tau) \neq \Phi_{S}(\tau)$ and (4.42) holds for $y \in \Phi_{S}(\tau)$. Since $\Phi_{\theta}(\tau) \equiv$ $\Phi_{S}(\tau)$ on $E^{o}$ by Lemma 4.4, we conclude that $\Phi_{\theta}(\tau) \neq \Phi(\tau)$ on $E^{o}$ and (4.42) holds for $y \in$ $\Phi_{\theta}(\tau)$. So (4.3) holds for $y \in \Phi_{\theta}(\tau)$ by (4.42). Then, we have that $\tau$ is in Case II with respect to $(\theta, K) \in S$ with $\theta \in E^{o}$ by Theorem 4.11 .

\section{Acknowledgments}

This work was supported by the NSF of Shandong Province (Grant Y2008A02) and the IIFSDU (Grant 2010ZRJQ002).

\section{References}

[1] H. Weyl, “Über gewöhnliche Differentialgleichungen mit Singularitäten und die zugehörigen Entwicklungen," Mathematische Annalen, vol. 68, no. 2, pp. 220-269, 1910.

[2] S. Clark and P. Zemánek, "On a Weyl-Titchmarsh theory for discrete symplectic systems on a half line," Applied Mathematics and Computation, vol. 217, no. 7, pp. 2952-2976, 2010.

[3] M. S. Eastham, "The limit- $2 n$ case of symmetric differential operators of order $2 n$," Proceedings of the London Mathematical Society, vol. 38, no. 3, pp. 272-294, 1979. 
[4] D. B. Hinton and J. K. Shaw, "On Titchmarsh-Weyl $M(\lambda)$-fuctions for linear Hamiltonian systems," Journal of Differential Equations, vol. 40, no. 3, pp. 316-342, 1981.

[5] D. B. Hinton and J. K. Shaw, "Hamiltonian systems of limit point or limit circle type with both endpoints singular," Journal of Differential Equations, vol. 50, no. 3, pp. 444-464, 1983.

[6] D. B. Hinton and J. K. Shaw, "On boundary value problems for Hamiltonian systems with two singular points," SIAM Journal on Mathematical Analysis, vol. 15, no. 2, pp. 272-286, 1984.

[7] A. M. Krall, " $M(\lambda)$ theory for singular Hamiltonian systems with one singular point," SIAM Journal on Mathematical Analysis, vol. 20, no. 3, pp. 664-700, 1989.

[8] A. M. Krall, " $M(\lambda)$ theory for singular Hamiltonian systems with two singular points," SIAM Journal on Mathematical Analysis, vol. 20, no. 3, pp. 701-715, 1989.

[9] J. Weidmann, Spectral Theory of Ordinary Differential Operators, vol. 1258 of Lecture Notes in Mathematics, Springer, Berlin, Germany, 1987.

[10] A. Zettl, Sturm-Liouville Theory, vol. 121 of Mathematical Surveys and Monographs, American Mathematical Society, Providence, RI, USA, 2005.

[11] A. R. Sims, "Secondary conditions for linear differential operators of the second order," vol. 6, pp. 247-285, 1957.

[12] B. M. Brown, D. K. R. McCormack, W. D. Evans, and M. Plum, "On the spectrum of second-order differential operators with complex coefficients," Proceedings of the Royal Society A, vol. 455, no. 1984, pp. 1235-1257, 1999.

[13] F. V. Atkinson, Discrete and Continuous Boundary Value Problems, Academic Press, New York, NY, USA, 1964.

[14] W. N. Everitt, M. Giertz, and J. B. McLeod, "On the strong and weak limit-point classfication of second-order differential expressions," Proceedings of the London Mathematical Society, vol. 29, no. 3, pp. 142-158, 1974.

[15] B. M. Brown and M. Marletta, "Spectral inclusion and spectral exactness for singular non-self-adjoint Sturm-Liouville problems," Proceedings of the Royal Society A, vol. 457, no. 2005, pp. 117-139, 2001.

[16] Y. Xiao, "Periodic solutions with prescribed minimal period for the second order Hamiltonian systems with even potentials," Acta Mathematica Sinica, vol. 26, pp. 49-76, 2010.

[17] W. N. Everitt and M. Giertz, "On integrable-square classfication of ordingary differential expressions," Journal of the London Mathematical Society, vol. 14, no. 2, pp. 41-45, 1964.

[18] W. N. Everitt, "On the limit-point classification of second-order differential operators," Journal of the London Mathematical Society, vol. 41, pp. 531-534, 1966.

[19] W. N. Everitt and M. Giertz, “On some properties of the domains of powers of certain differential operators," Proceedings of the London Mathematical Society, vol. 24, no. 3, pp. 756-768, 1972.

[20] W. N. Everitt, D. B. Hinton, and J. S. Wong, "On the SLP condition of ordinary differential equations of order 2n," Proceedings of the London Mathematical Society, vol. 29, no. 3, pp. 351-357, 1974.

[21] J. Qi and S. Chen, "Strong limit-point classification of singular Hamiltonian expressions with complex coefficients," Proceedings of the American Mathematical Society, vol. 132, no. 6, pp. 1667-1674, 2004.

[22] J. Qi and H. Wu, "Limit point, strong limit point and Dirichlet conditions for Hamiltonian differential systems," Mathematische Nachrichten, vol. 284, pp. 764-780, 2011.

[23] H. Sun and J. Qi, "On classification of second-order differential equations with complex coefficients," Journal of Mathematical Analysis and Applications, vol. 372, no. 2, pp. 585-597, 2010.

[24] D. E. Edmunds and W. D. Evans, Spectral Theory and Differential Operators, Clarendon Press, Oxford, UK, 1987. 


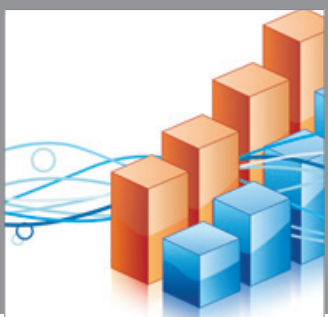

Advances in

Operations Research

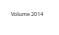

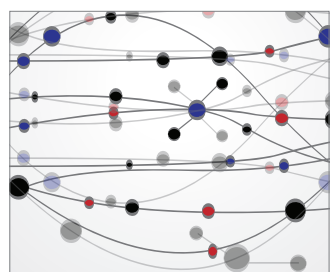

\section{The Scientific} World Journal
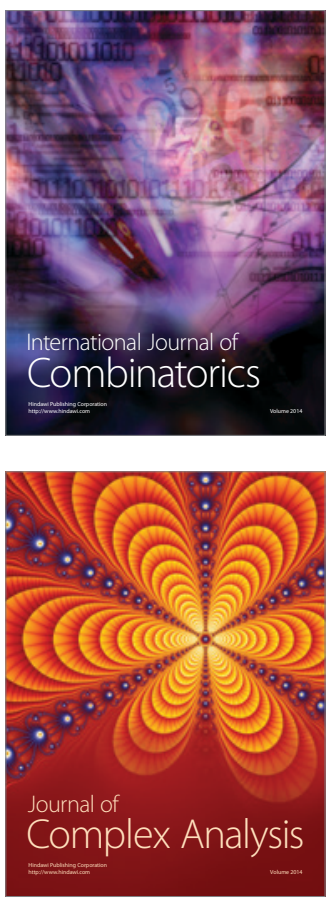

International Journal of

Mathematics and

Mathematical

Sciences
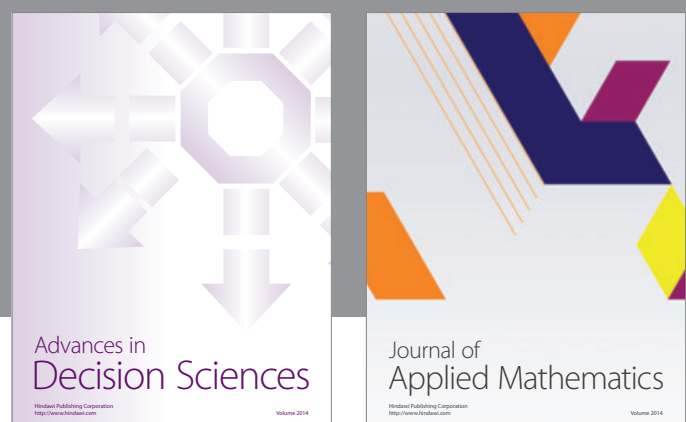

Journal of

Applied Mathematics
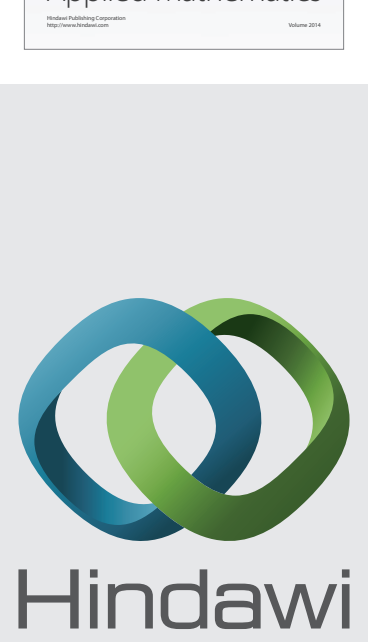

Submit your manuscripts at http://www.hindawi.com
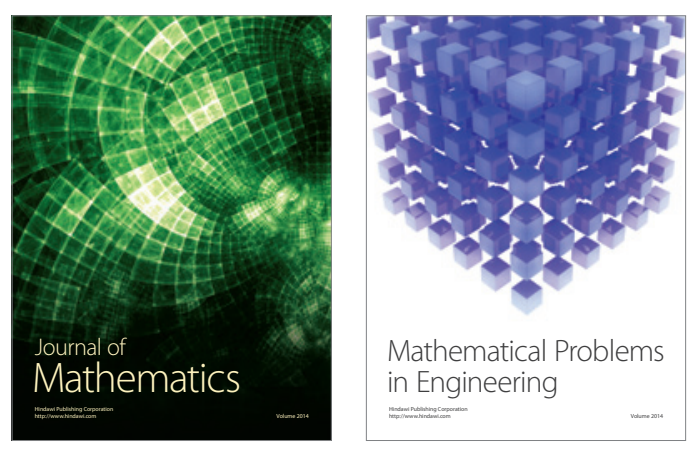

Mathematical Problems in Engineering
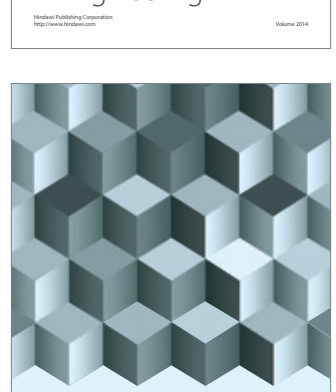

Journal of

Function Spaces
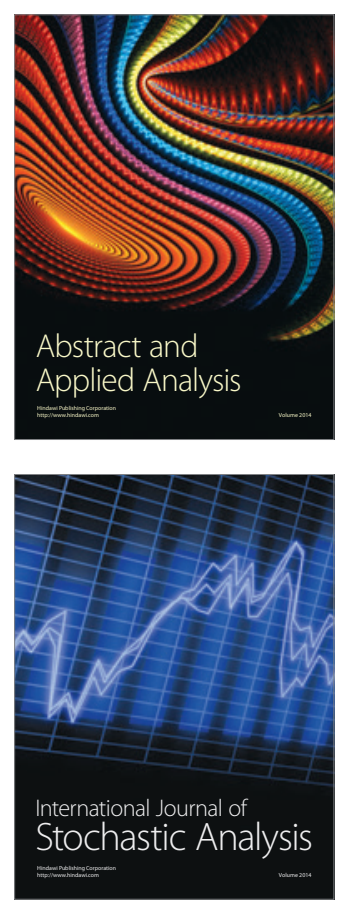

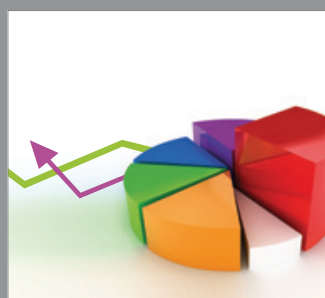

ournal of

Probability and Statistics

Promensencen
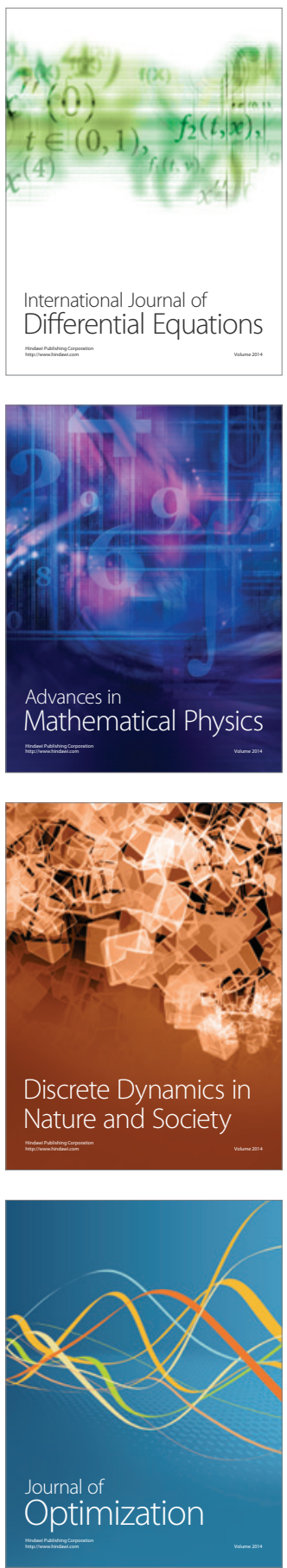